\title{
ПОСТРОЕНИЕ ПОЛНЫХ КОНТРОЛИРУЮЩИХ ТЕСТОВ КОМБИНАЦИОННЫХ СХЕМ
}

\author{
(Представил Э. Тьсугу)
}

\section{1. Введение}

Трудности при составлении тестов для интегральных цифровых схем с нерегулярной структурой обусловлены прежде всего необходимостью учета всех возможных кратных неисправностей. Известны методы синтеза тестов комбинационных схем (KC), избегающие перечисления всех возможных сочетаний кратных неисправностей, но в то же время косвенно учитывающие их $\left[{ }^{1-6}\right]$. С определенным успехом эти методы можно обобщить и на схемы с памятью.

В $\left[{ }^{1-3}\right]$ предложены аналитические методы построения проверяющих тестов, требующие представления схемы в эквивалентной нормальной форме $\left[{ }^{1-2}\right]$ или в виде эквивалентного дерева $\left[{ }^{3}\right]$. Недостатками этих подходов являются громоздкость модели, а также возможность получения избыточных тестов из-за избыточности модели. Структурная модель схемы в $\left[{ }^{4}\right]$ предусматривает двухэтапный синтез тестов сначала построение полного диагностического теста, а затем его редукцию до полного проверяющего теста, что также делает метод трудоемким в применении.

В $\left[{ }^{5,6}\right]$ используется метод проверки исправности пути КС с помощью пар наборов, обнаруживающих неисправности «константа 0» и «константа 1》 элементов пути, причем эти наборы являются соседними по переменной, приписанной входному полюсу данного пути. В [ $\left.{ }^{5}\right]$ этот метод реализован для структурной модели объекта, в $\left[{ }^{6}\right]-$ для аналитической модели в виде скобочной формы. Однако он неприемлем, если условия существования парных тестов, обладающих свойством соседства, не выполняются.

Дальнейшее развитие метод парных тестов получил в $\left[{ }^{6}\right]$, где дается методика вычисления дополнительных наборов к паре тестов, если последняя недоста́точна для проверки конкретного пути. Методу присуща определенная сложность, так как он использует модель объекта в виде скобочной формы и требует вычисления дополнительных наборов отдельно для всех повторяющихся букв в выражении функции.

Ниже излагается метод построения полного проверяющего теста $\mathrm{KC}$, представленной в виде системы альтернативных графов (АГ) [ [ $]$, c использованием понятий и определений также из [7]. Метод базируется на понятии групповых тестов, синтез которых алгоритмически проще, чем вычисление дополнительных наборов [ $\left.{ }^{6}\right]$.

\section{2. Определения}

Для простоты изложения рассмотрим одновыходные $\mathrm{KC} \Phi=\{Z, F\}$, в которых допускаются разветвления во входных узлах и не допускают- 
'Рис. 1.
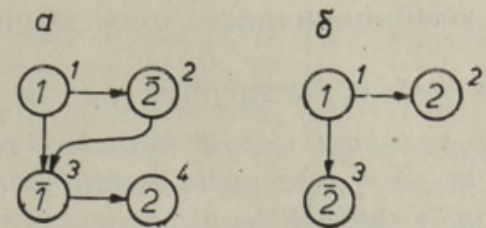

ся во внутренних. Для их задания достаточно одной функции $y=f(X)$, где $f \in F, y \cup X=Z \cdot\left[{ }^{7}\right]$. На возможности обобщения получаемых результатов для общего случая $\mathrm{KC}$, где $\left|F_{\text {вн }}\right|>0$ и $\left|F_{\text {вых }}\right|>1$, остановимся ниже.

Представим рассматриваемые КС в виде АГ $G=(M, \Gamma)$. Согласно интерпретации АГ, каждой вершине $m \in M$ соответствует некоторая ветвь в реальной схеме. Следовательно, класс кратных константных неисправностей на ветвях схемы $\left[{ }^{1-6}\right]$ можно заменить моделью кратных константных неисправностей вершин $m \in M: e(m) \equiv \alpha, \alpha \in\{0,1\}$.

Введем следующие определения.

АГ называется канонически м, если для всех вершин $m_{i} \in M$, где $\left|\Gamma m_{i}\right|=2$, выполняется одно из двух возможных отношений $m_{i}^{\alpha} \in \hat{\Gamma}\left(m_{i}^{\bar{\alpha}}\right), \alpha \in\{0,1\}$. Например, граф на рис. $1, a-$ канонический, а граф на рис. $1, \sigma-$ нет. Оба графа соответствуют функции $y=z_{1} \bar{z}_{2} \bigvee \bar{z}_{1} z_{2}$. Алгоритм синтеза канонических АГ для заданной КС приведен в $\left[^{8}\right]$. Далее рассматриваются только канонические АГ.

Тест для проверки неисправности $e\left(m_{i}\right) \equiv \bar{\alpha}, \alpha \in\{0,1\}$, в вершине $m_{i} \in M$ будем обозначать через $E_{\alpha}\left(m_{i}\right)$. Он представляет собой набор значений входных переменных $z \in X$, активизирующий какие-то два пути $l\left(m_{0}, \alpha\right)$ и $l\left(m_{i}^{\bar{\alpha}}, \bar{\alpha}\right)$ так, чтобы выполнялись условия

$$
\begin{aligned}
& G\left(m_{0}\right)=\alpha, \\
& G\left(m_{i}^{\bar{\alpha}}\right)=\bar{\alpha},
\end{aligned}
$$

где $\Gamma^{-1} m_{0}=\varnothing$. В случае неисправности $r_{\bar{\alpha}}$ значение $G\left(m_{0}\right)$ в силу

(2) изменяется

$$
E_{\alpha}\left(m_{i}\right) \& r_{\bar{\alpha}} \rightarrow G\left(m_{0}\right)=\bar{\alpha}
$$

указывая тем самым на наличие неисправности.

П а рный тест для проверки обеих неисправностей $e\left(m_{i}\right) \equiv \alpha$, $\alpha=\{0,1\}$, будем обозначать через $E\left(m_{i}\right)=\left\{E_{0}\left(m_{i}\right), E_{1}\left(m_{i}\right)\right\}$. Значения переменных $z \in X \backslash V_{Z}\left(m_{i}\right)$ для обоих наборов $E_{\alpha}\left(m_{i}\right) \in E\left(m_{i}\right)$ должны быть одинаковыми.

Множество проверенных вершин $M_{\text {п }} \subseteq M-$ это вершины графа, тесты для проверки которых построены до текущего шага синтеза.

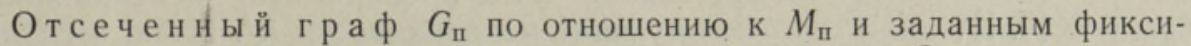
рованным значениям переменных $z \in X-$ это граф $G$, из которого удалены все дуги, соответствующие отображению $\Gamma(m, \overline{e(m)}), m \in M_{\text {п. }}$.

Путь $l\left(m_{i}, \varrho\right)$, где $\varrho \in\left\{0,1, m_{j}\right\}, m_{i}, m_{j} \in M$, называется блоки ров анным от заданной вершины $m_{k} \in M \backslash\left\{m_{i}, m_{j}\right\}$, если в отсеченном графе $G_{\text {п }}$ не существует пути $l^{\prime}\left(m^{\prime}, \rho^{\prime}\right)$ такого, что $m_{k} \in l^{\prime}, m^{\prime} \in l$ и $\mathrm{Q}^{\prime} \neq \mathrm{Q}$, где $\mathrm{Q}^{\prime} \in\{0,1\}$.

Вершины $m_{i}, m_{j} \in M$ называются подобными, если $V_{Z}\left(m_{i}\right)=$ $=V_{Z}\left(m_{j}\right)$ и $\beta\left(m_{i}\right)=\beta\left(m_{j}\right)$. 


\section{3. Проверка комбинационных схем парными тестами}

Метод синтеза парных тестов базируется на следующем утверждении. Т еорем а 1. Положительный исход парного теста $E\left(m_{i}\right)$ означает исправность вериины $m_{i} \in M$ при выполнении одного из двух условий: 1) в графе $G$ отсутствуют подобные $m_{i}$ вериины, или 2) пути, активизированные парным тестом $E\left(m_{i}\right)$, заблокированы от подобных вершин.

Д ок а з а ельство. Для проверки неисправности

$$
r_{\bar{\alpha}}: e\left(m_{i}\right) \equiv \bar{\alpha}
$$

$\alpha \in\{0,1\}$, предназначен тест $E_{\alpha} \in E\left(m_{i}\right)$

$$
\begin{aligned}
& E_{\alpha} \rightarrow G\left(m_{0}\right)=\alpha, \\
& E_{\alpha} \rightarrow G\left(m_{i}^{\bar{\alpha}}\right)=\bar{\alpha} \text {, }
\end{aligned}
$$

откуда в случае неисправности $r_{\bar{\alpha}}$ получим

$$
E_{\alpha} \& r_{\bar{\alpha}} \rightarrow G\left(m_{0}\right)=\bar{\alpha},
$$

и активизацию некоторого пути $l\left(m_{0}, \bar{\alpha}\right)$. Из-за определенного множества неисправностей $R^{\prime},\left|R^{\prime}\right|=1,2, \ldots$, возможны компенсация $r_{\bar{\alpha}}$, если

$$
E_{\alpha} \& R^{\prime} \rightarrow G\left(m_{k}\right)=\alpha,
$$

где

$$
m_{k} \in M_{\bar{\alpha}}(l)=\{m \mid m \in l, e(m)=\bar{\alpha}\} \subseteq M,
$$

и активизация пути $l^{\prime}\left(m_{k}, \alpha\right)$. В этом случае на основе выражений (4), (6) и (7) получим

$$
E_{\alpha} \bigvee E_{\alpha} \& r_{\bar{\alpha}} \& R^{\prime} \rightarrow G\left(m_{0}\right)=\alpha
$$

Следовательно, неисправность $r_{\bar{\alpha}}$ не будет выявлена тестом $E_{\alpha}$. Однако компенсирующие неисправности $R^{\prime}$ будут обнаружены тестом $E_{\bar{\alpha}} \in E\left(m_{i}\right)$, если выполняется условие

$$
E_{\bar{\alpha}} \& R^{\prime} \rightarrow G\left(m_{k}\right)=\alpha .
$$

Предположим обратное, что (9) не выполняется. Тогда должна существовать некоторая вершина $m_{j} \in l^{\prime}\left(m_{k}, \alpha\right)$, подобная $m_{i}$. Но это противоречит первому условию теоремы. С другой стороны, из-за блокировки (второе условие теоремы) не существует пути $l^{\prime}\left(m_{k}, \alpha\right)$ такого, чтобы $l^{\prime} \cap M^{\prime}\left(m_{i}\right) \neq \varnothing$, где $M^{\prime}\left(m_{i}\right)$ - множество подобных $m_{i}$ вершин. Следовательно, предположение неверно и выражение (9) при условиях теоремы всегда выполняется. Отсюда следует вывод, что парный тест $E\left(m_{i}\right)$ обязательно обнаруживает или проверяемую неисправность $r \frac{\bar{\alpha}}{,}, \alpha \in\{0,1\}$, или компенсирующие неисправности $R^{\prime}$. Итак, в случае положительного исхода парного теста $E\left(m_{i}\right)$ вершина $m_{i}$ исправна.

Из теоремы 1 вытекает следующий

А лгорит м:

Шаг 1. Определяется подмножество $M^{\prime} \subseteq M$ вершин, имеющих себе подобные.

Шаг 2. Если $M^{\prime}=M$, то синтез тестов по данному алгоритму невозможен (см. раздел 4). 
Шаг 3. Строятся тестовые пары для всех вершин $m \in M \backslash M^{\prime}$. Образуется подмножество $M_{\Pi \prime}=M \backslash M^{\prime}$.

Шаг 4. Если $M_{n}=M$, то конец (тесты построены).

Шаг 5. Находится $m_{i} \in M \backslash M_{\Perp}$, для которой при построении $E\left(m_{i}\right)$ выполняются условия блокировки путей от $m_{j} \in M^{\prime}\left(m_{i}\right) . M_{\mathrm{u}}$ доопределяется вершиной $m_{i}$. Переход к 4-му шагу.

Шаг 6. Если 5-й шаг не выполняется из-за отсутствия нужной $m_{i}$, то конец (синтез тестов для вершин $m_{i} \in M \backslash M_{\text {I }}$ по данному алгоритму невозможен).

Обобщение теоремы 1 и алгөритма на общий случай $\mathrm{KC}$ с внутренними разветвлениями $\left(F_{\text {вн }} \neq \varnothing\right)\left[{ }^{7}\right]$ не представляет труда. Для этого

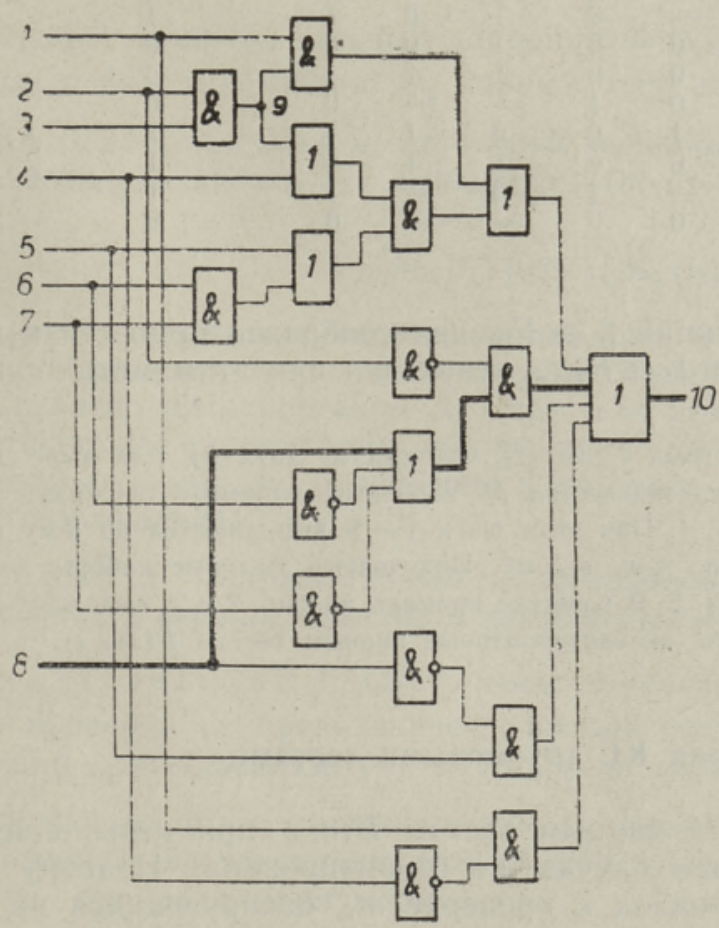

Рис. 2.

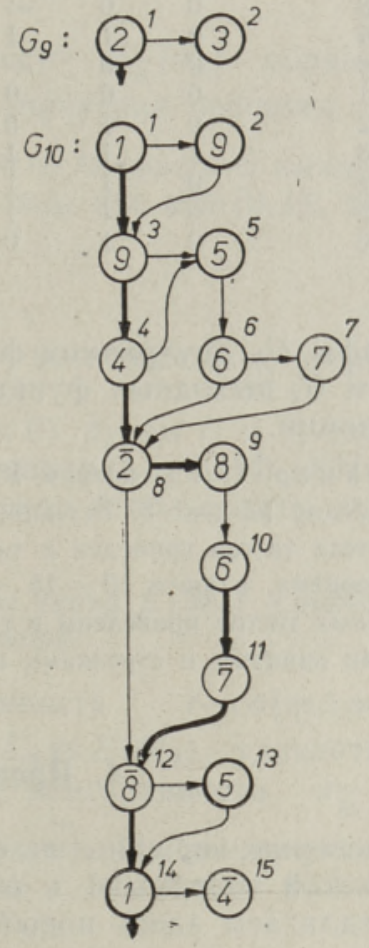

Рис. 3.

Таблица 1

\begin{tabular}{c|c|c|c}
\multirow{2}{*}{ Шаг } & \multicolumn{2}{|c|}{ Номер набора } & \multirow{2}{*}{ Проверяемая вершин } \\
\cline { 2 - 3 } & $E_{1}(m)$ & $E_{0}(m)$ & \\
1 & & & $m_{10,4}$ \\
2 & 1 & 2 & $m_{10,15}$ \\
3 & 4 & 3 & $m_{10,6}$ \\
4 & 5 & 6 & $m_{10,10}$ \\
5 & 8 & 7 & $m_{10,7}$ \\
6 & 5 & 9 & $m_{10,11}$ \\
7 & 10 & 7 & $m_{10,9}$ \\
8 & 11 & 7 & $m_{10,12}$ \\
9 & 12 & 2 & $m_{10,8}$ \\
10 & 11 & 2 & $m_{10,1}$ \\
11 & 13 & 6 & $m_{9,2}, m_{10,2}$ \\
12 & 13 & 3 & $m_{9,1}, m_{10,3}$ \\
13 & 14 & 7 & $m_{10,5}$ \\
14 & 15 & 6 & $m_{10,13}$ \\
15 & 12 & 16 & $m_{10,14}$
\end{tabular}




\begin{tabular}{l|lllllllll|lll}
\hline $\begin{array}{l}\text { Номер } \\
\text { набора }\end{array}$ & $z_{1}$ & $z_{2}$ & $z_{3}$ & $z_{4}$ & $z_{5}$ & $z_{6}$ & $z_{7}$ & $z_{8}$ & & $z_{9}$ & $z_{10}$ \\
\hline
\end{tabular}

$\begin{array}{rllllllllll}1 & 0 & 1 & 0 & 1 & 1 & - & - & 1 & 0 & 1 \\ 2 & 0 & 1 & 0 & 0 & 1 & - & - & 1 & 0 & 0 \\ 3 & 1 & 1 & 0 & 1 & 0 & 0 & - & 1 & 0 & 0 \\ 4 & 1 & 1 & 0 & 0 & 0 & 0 & - & 1 & 0 & 1 \\ 5 & 0 & 1 & 1 & - & 0 & 1 & 1 & 1 & 1 & 1 \\ 6 & 0 & 1 & 1 & 1 & 0 & 0 & 1 & 1 & 1 & 0 \\ 7 & 0 & 0 & 1 & 0 & 0 & 1 & 1 & 0 & 0 & 0 \\ 8 & 0 & 0 & - & 0 & 0 & 0 & 1 & 0 & 0 & 1 \\ 9 & 0 & 1 & 1 & - & 0 & 1 & 0 & 1 & 1 & 0 \\ 10 & 0 & 0 & - & 0 & 0 & 1 & 0 & 0 & 0 & 1 \\ 11 & 0 & 0 & 0 & 0 & 0 & 1 & 1 & 1 & 0 & 1 \\ 12 & 0 & 1 & 0 & 0 & 1 & - & - & 0 & 0 & 1 \\ 13 & 1 & 1 & 1 & 1 & 0 & 0 & 1 & 1 & 1 & 1 \\ 14 & 0 & 1 & 1 & 0 & 0 & 1 & 1 & 0 & 1 & 1 \\ 15 & 0 & 1 & 1 & - & 1 & 0 & - & 1 & 1 & 1 \\ 16 & 0 & 1 & 0 & 0 & 0 & - & - & 0 & 0 & 0\end{array}$

графы $G_{k}$ внутренних функций $f_{k} \in F_{\text {вн }}$ целесообразно привести к графам $G_{i}$ выходных функций $f_{i} \in F_{\text {вых }}$, используя при этом метод суперпозиции.

Приме р 1. Рассмотрим КС (рис. 2) из [']. Система из двух АГ для этой схемы показана на рис. 3 . Вершины из множества $M^{\prime}$ выделены жирными кружками. Ход синтеза тестов приведен в табл. 1. При этом шаги $1-9$ выполняются по 3-му шагу алгоритма, а шаги $10-15$ - по 4-му и 5-му. Полученные тестовые наборы для 15 парных тестов приведены в табл. 2. В качестве примера на рис. 2 и 3 выделены жирными линиями и стрелками пути, активизированные парным тестом $E\left(m_{10}, 9\right)$.

\section{4. Проверка КС групповыми тестами}

Проверка вершины $m_{i} \in M$ парным-тестом $E\left(m_{i}\right)$ при невыполнении условий теоремы 1 в общем случае не гарантирована. Поэтому мы предлагаем здесь новый подход к проверке $m_{i}$, базирующийся на понятии $\alpha$-групп и групповых тестов.

Назовем $\alpha$-г у ппой, $\alpha \in\{0,1\}$, подмножество вершин $M_{\alpha}(l)$ на некотором активизированном пути $l=l\left(m_{0}, \alpha\right)$

$$
M_{\alpha}(l)=\left\{m_{i} \mid m_{i} \in l, e\left(m_{i}\right)=\alpha\right\} \subseteq M,
$$

где

$$
\forall m_{i}, m_{j} \in M_{\alpha}(l), \quad i \neq j: V_{Z}\left(m_{i}\right) \not \equiv V_{Z}\left(m_{j}\right) .
$$

Согласно интерпретации АГ, подмножеству $M_{0}$ (или $M_{1}$ ) соответствует некоторая элементарная дизъюнкция или конъюнкция, не содержащая одинаковых переменных и входящая соответственно в конъюнктивную нормальную форму (КНФ) или дизъюнктивную нормальную форму (ДНФ) функции, представленной графом $G \cdot$ Например, для графа на рис. $1, a$ вершины 1,4 могут составлять 0 -группу $M_{0}$, а вершины $1,2-$ 1 -группу $M_{1}$. Из-за невыполнения (10) вершины 1,3 и 2,4 не могут образовывать $\alpha$-группу.

Групповой тест $E\left(M_{\alpha}\right)$ для проверки вершин $m_{i} \in M_{\alpha}$ некоторой $\alpha$-группы $M_{\alpha}$ представляет собой $\left|M_{\alpha}\right|$ парных тестов, в наборах $E\left(M_{\alpha}\right)$ которых значения переменных $z \in Z \backslash V_{Z}\left(M_{\alpha}\right)$ одинаковы.

Введем обозначение 


$$
V_{Z}\left(M_{\alpha}\right)=\bigcup_{m_{t} \in M_{\alpha}} V_{Z}\left(m_{i}\right)
$$

Групповой тест $E\left(M_{\alpha}\right)=\left\{E_{\alpha}\left(M_{\alpha}\right),\left\{E_{\bar{\alpha}}\left(m_{i}\right)\right\}\right\}$ состоит из базового теста $E_{\alpha}$, где

$$
\forall m_{i} \in M_{\alpha}: e\left(m_{i}\right)=\alpha,
$$

и из $\left|M_{\alpha}\right|$ дополнительных тестов $E_{\bar{\alpha}}\left(m_{i}\right)$, где

$$
\begin{aligned}
& e\left(m_{i}\right)=\bar{\alpha}, \\
& \forall m_{j} \in M_{\alpha} \backslash m_{i}: e\left(m_{j}\right)=\alpha
\end{aligned}
$$

для любой $m_{i} \in M_{\alpha}$. Каждый дополнительный тест $E_{\bar{\alpha}}\left(m_{i}\right)$ образует вместе с базовым тестом $E_{\alpha}$ парный тест для проверки вершины $m_{i}$.

О п ределен и е. Если из некоторой вершины $m \in M$ графа $G$ выходят два несовпадающих пути $l_{1}(m, \alpha)$ и $l_{2}(m, \alpha), l_{1} \neq \equiv l_{2}$, где $\alpha \in\{0,1\}$, так, что

$$
V_{Z}\left[M_{\alpha}\left(l_{1}\right)\right] \subseteq V_{Z}\left[M_{\alpha}\left(l_{2}\right)\right],
$$

то путь $l_{1}$ называется поглощающим, путь $l_{2}-$ поглощаемым, а граф $G$ - избыточным (соответствует избыточной $\mathrm{KC}$ ).

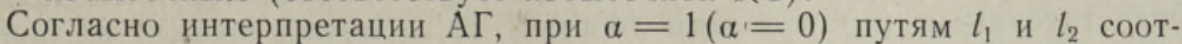
ветствуют две элементарные конъюнкции (дизъюнкции) в ДНФ (КНФ) булевой функции, по отношению к которым можно использовать правило поглощения.

Т ео рем а 2. Положительный исход группового теста $E\left(M_{\alpha}\right)$ означает исправность вериин $m_{i} \in M_{\alpha}$ в неизбыточном графе.

Доказательство. 1. Пусть имеется неисправность $\quad r_{\bar{\alpha}}: e\left(m_{i}\right) \equiv \bar{\alpha}$. Для проверки ее предназначен базовый тест $E_{\alpha} \in E\left(M_{\alpha}\right)$, удовлетворяющий соотношениям (4) и (5), а в случае неисправности $r_{\bar{\alpha}}$ соотношению (6) с активизированием пути $l\left(m_{0}, \bar{\alpha}\right)$. Из-за определенного множества неисправностей $R^{\prime},\left|R^{\prime}\right|=1,2, \ldots$, возможны компенсация $r_{\bar{\alpha}}$, если имеет место

$$
E_{\alpha} \& R^{\prime} \rightarrow G\left(m_{k}\right)=\alpha,
$$

где $m_{k} \in M_{-\alpha}(l), \quad$ и активизация некоторого пути $l^{\prime}\left(m_{k}, \alpha\right)$. Здесь $M_{\bar{\alpha}}(l)-\bar{\alpha}$-группа пути $l\left(m_{0}, \bar{\alpha}\right)$. Условием обнаружения компенсирующих неисправностей тестом $E_{\bar{\alpha}}\left(m_{i}\right) \in E\left(M_{\alpha}\right)$ будет аналогичное (9) отношение

$$
E_{\bar{\alpha}}\left(m_{i}\right) \& R^{\prime} \rightarrow G\left(m_{k}\right)=\alpha
$$

Но поскольку в общем случае возможно наличие вершины $m_{j} \in l^{\prime}\left(m_{k}, \alpha\right)$, подобной $m_{i}$, то условие (12) может не выполняться.

Выбираем вершину $m_{p} \in M_{\alpha}$ такую, чтобы $V_{Z}\left(m_{p}\right) \notin V_{Z}\left(l^{\prime}\left(m_{k}, \alpha\right)\right)$. Если такой вершины не существует, то, согласно определению, граф избыточен, что противоречит предположению. Но для выбранной $m_{p}$ при выполнении (11) всегда имеет место

$$
E_{\bar{\alpha}}\left(m_{p}\right) \& R^{\prime} \rightarrow G\left(m_{p}\right)=\alpha,
$$

так как для $m_{j}$ будет 


$$
\dot{E}_{\bar{\alpha}}\left(m_{p}\right) \rightarrow e\left(m_{j}\right) \doteq \alpha .
$$

Следовательно, дополнительный тест $E_{\bar{\alpha}}\left(m_{p}\right)$ для проверки вершины $m_{p}$ обнаруживает неисправности $R^{\prime}$, мешающие проверке вершины $m_{i}$ тестами $E_{\alpha}\left(M_{\alpha}\right)$ и $E_{\bar{\alpha}}\left(m_{i}\right)$.

2. Пусть имеется неисправность $r_{\alpha}: e\left(m_{i}\right) \equiv \alpha$. Для проверки ее предназначен дополнительный тест $E_{\bar{\alpha}}\left(m_{i}\right) \in E\left(M_{\alpha}\right)$ :

$$
\begin{aligned}
& E_{\bar{\alpha}}\left(m_{i}\right) \rightarrow G\left(m_{0}\right)=\bar{\alpha}, \\
& E_{\bar{\alpha}}\left(m_{i}\right) \rightarrow G\left(m_{i}^{\alpha}\right)=\alpha .
\end{aligned}
$$

Привести к компенсации $r_{\alpha}$ могут только неисправности $r^{\prime} \bar{\alpha}: e\left(m_{j}\right) \equiv \bar{\alpha}$, $m_{j} \in M_{\alpha}$, из-за которых не выполняется (16). Но эти неисправности (или компенсирующие их неисправности) обнаруживаются, согласно первой части доказательства, хотя бы одним из наборов $E\left(M_{\alpha}\right)$.

Обобщая доказательство на все $m_{i} \in M_{\alpha}$, приходим к выводу, что при положительном исходе группового теста $E\left(M_{\alpha}\right)$ вершины $m_{i} \in M_{\alpha}$ исправны, а при отрицательном исходе хотя бы одного из наборов $E\left(M_{\alpha}\right)$ судить о их состоянии нельзя.

Доопределяя алгоритм дополнительной процедурой синтеза групповых тестов для вершин $m_{i} \in M^{\prime}$, для которых парные тесты оказались недостаточными (по условиям теоремы 1), получим полный алгоритм синтеза тестов для неизбыточных КС. Обобщить результаты на КС, у которых $F_{\text {вн }} \neq \varnothing$, позволяет применение метода суперпозиции гра-

\begin{tabular}{|c|c|c|c|c|c|}
\hline \multirow{2}{*}{ Шаг } & \multirow{2}{*}{ Группа } & \multicolumn{3}{|c|}{ Номера наборов } & \multirow{2}{*}{$\begin{array}{c}\text { Проверяемые } \\
\text { вершины }\end{array}$} \\
\hline & & $E_{\bar{\alpha}}$ & $E_{\alpha}^{\prime}$ & $E_{\alpha}^{\prime \prime}$ & \\
\hline
\end{tabular}
фов.

Таблица 3

$\begin{array}{llllll}1 & M_{0}{ }^{1} & 1 & 2 & 3 & m_{1}, m_{3} \\ 2 & M_{0}^{2} & 4 & 5 & 6 & m_{2}, m_{4} \\ 3 & M_{0}^{3} & 7 & 3 & 5 & m_{5}, m_{7} \\ 4 & M_{0}^{4} & 8 & 2 & 6 & m_{6}, m_{8}\end{array}$

Таблица 4

\begin{tabular}{l|llllll}
\hline $\begin{array}{l}\text { Номер } \\
\text { набора }\end{array}$ & $z_{1}$ & $z_{2}$ & $z_{3}$ & $z_{4}$ & $z_{5}$ \\
\hline
\end{tabular}

$\begin{array}{llllll}1 & 0 & 1 & 0 & 1 & 0 \\ 2 & 1 & 1 & 0 & 1 & 1 \\ 3 & 0 & 1 & 1 & 1 & 1 \\ 4 & 1 & 0 & 1 & 0 & 0 \\ 5 & 1 & 0 & 1 & 1 & 1 \\ 6 & 1 & 1 & 1 & 0 & 1 \\ 7 & 0 & 0 & 1 & 1 & 0 \\ 8 & 1 & 1 & 0 & 0 & 0\end{array}$

Приме р 2. Рассмотрим КС (рис. 4) из $\left[^{5}\right]$ и ее АГ (рис. 5). Легко заметить, что алгоритм неприемлем ни для одной вершины, так как $M^{\prime}=M$. Выделим в АГ четыре 0 -группы $M_{0} i, i=\overline{1,4}$ (обведены на рис. 5 пунктиром). Ход синтеза групповых тестов поясняет табл. 3 , а полученные тестовые наборы приведены в табл. 4. Тест для проверки группы $M_{0}^{2}$ показан на рис. 5 жирными стрелками и кружками. 


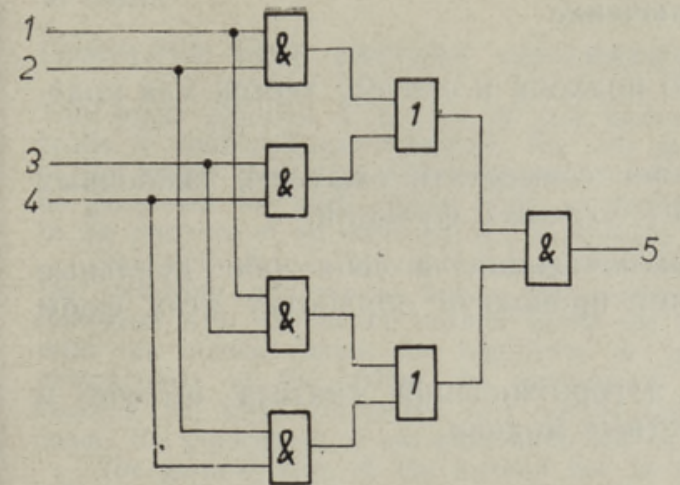

Рис. 4.

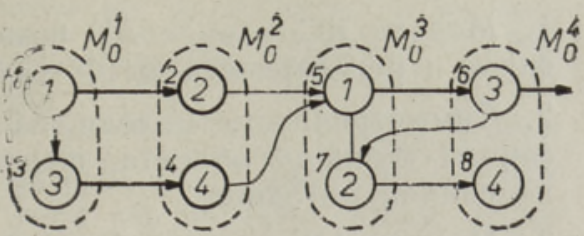

Рис. 5.

\section{5. Проверка избыточных КС}

До сих пор рассматривались неизбыточные КС, описываемые неизбыточными АГ. Легко убедиться в том, что теорема 2 неверна в общем случае для избыточных КС (избыточных АГ).

Рассмотрим в качестве примера избыточную КС на рис.. 6 и соответствующий ей $\mathrm{AГ}$ на рис. 7. Пусть имеются неисправности $e\left(m_{3}\right) \equiv 0$ и $e\left(m_{4}\right) \equiv 1$. Поскольку у вершины $m_{3}$ есть ей подобная $\left(m_{6}\right)$, то парный тест ее не проверяет. Легко также убедиться, что групповой тест для $M_{1}^{\prime}=\left\{m_{1}, m_{3}\right\}$ приводит к положительному исходу, хотя одна из проверяемых вершин неисправна. Но все же имеется группа $M^{\prime \prime}{ }_{1}=$ $=\left\{m_{2}, m_{3}\right\}$, групповой тест которой проверяет вершину $m_{3}$.

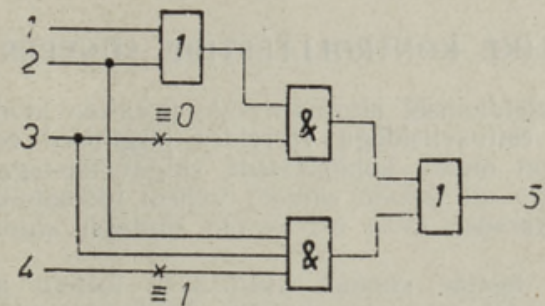

Рис. 6.

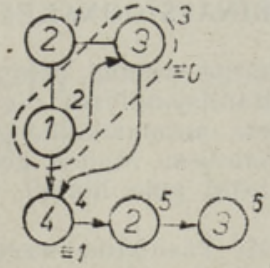

Рис. 7.

Те орем а 3. Положительный исход группового теста $E\left[M_{\alpha}(l)\right]$ означает исправность вериин $m_{i} \in M_{\alpha}(l)$, если путь $l$ в графе $G$ не поглощзает ни одного другого пути.

Теорема 3 усиливает теорему 2 на случай избыточных АГ. Доказательство ее вытекает также из доказательства теоремы 2. Так, например, на рис. $7 \alpha$-группа $M^{\prime \prime}{ }_{1}=\left\{m_{2}, m_{3}\right\}$ соответствует пути, который не поглощает других. Этого нельзя сказать об $\alpha$-группе $M_{1}^{\prime}=\left\{m_{1}, m_{3}\right\}$.

Из теоремы 3 вытекает следующее доопределение к вышеприведенному алгоритму: при формировании $\alpha$-группы $M_{\alpha}(l)$ для проверки вершины $m_{i}$ групповым тестом необходимо выбирать путь $l$, не поглощающий других путей.

Легко убедиться, что если для некоторой вершины $m_{i}$ все проходящие через нее пути оказываются поглощающими, то необнаруженная неисправность $e\left(m_{i}\right)=\bar{\alpha}, m_{i} \in M_{\alpha}$, несущественна - она снижает избыточность объекта, но не изменяет его функцию. 


\section{Заключение}

Перечислим преимущества данного подхода к синтезу тестов для кратных неисправностей $\mathrm{KC}$ :

1. Модель КС в виде АГ позволяет совместить сжатость скобочных форм и регулярность ДНФ (КНФ) булевых функций.

2. Симметричность модели АГ, включающая в себя обе дуальные формы функции объекта, позволяет проводить обработку этих форм по единой методике.

3. Модель АГ более проста для алгоритмизацйи синтеза $\alpha$-групп и групповых тестов, чем другие известные модели.

\section{ЛИТЕРАТУ РА}

1. Ч и пули с В. П., Автоматика и телемеханика, № 11, 159-163 (1971).

2. Ч и пули с В. П., Автоматика и телемеханика, № 9, 165-172 (1973).

3. Д у б р о Э. Б., Автоматика и телемеханика, № 1, 154-161 (1975).

4. К а р а в а й М. Ф., Автоматика и телемеханика, № 1, 162-170 (1975).

5. Би ргер А. Г., Гу р в и Е. Т., Кузнецов С. С., Автоматика и телемеханика, № 8, 113-120 (1975).

6. К ог а Н И. В., Автоматика и вычисл. техн., № 2, 31-37 (1976).

7. Л х у а р у Т., Палль М., У б а р Р., Изв. АН ЭССР, Физ. Матем., (в печати).

8. У 6 а р Р. Р., Тр. Таллин, политехн. ин-та, № 409, 75-81 (1976).

Таллинский политехнический институт
Поступила в редакцию 12/V 1982

\section{R. UBAR}

\section{KOMBINATSIOONSKEEMIDE TÄIELIKE KONTROLLTESTIDE SONTEES}

Artiklis esitatud meetod põhineb objekti kirjeldamisel alternatiivsete graafide mudelina, millel on traditsiooniliste digitaalskeemide esitusviisidega võrreldes järgmised eelised: disjunktiivsete normaalkujudega võrreldes on mudel kompaktsem, sulgavaldistega vôrreldes regulaarsem. Mudeli kompaktsuse tōttu on testide süntees vähem töömahukas ja saadavad testid pole liiased. Mudeli regulaarsuse tõttu on testide sünteesi algoritmid lihtsamad.

Vastavalt käsitletud meetodile toimub testide süntees üksikrikete loetelu põhjal, kordsete rikete kombinatsioonide loetlemine pole seejuures tarvilik. Sünteesitavad testid aga avastavad peale üksikrikete ka vōimalikke kordseid rikkeid. Iga signaalitee korrasoleku kontrolliks sünteesitakse kahest testist koosnev testipaar. On määratud tingimused, millal testipaarid pole piisavad. Nendel juhtudel tuleb sünteesida kolmest või enamast testist koosnevad testigrupid, mis võimaldavad kindlaks teha teatava grupi signaaliteede korrasoleku. On esitatud testipaaride ja -gruppide sünteesi metoodika ja tõestatud teoreemid, mis formuleerivad testide täielikkuse tingimused. 


\section{R. $U \hat{B} A \hat{R}$}

\section{COMPLETE TEST PATTERN GENERATION FOR COMBINATIONAL NETWORKS}

This paper presents a method of test pattern generation to detect arbitrary multiple faults in combinational networks. The test generation will be carried out on the basis of the single fault list. The enumeration of multiple faults is not needed. A test will be generated for each single fault, so that this fault could be detected by the test in the presence of an arbitrary set of multiple faults.

The method uses alternative graph models tor describing the given object. It gives the following advantages before the traditional ways of describing digital circuits: compared with disjunctive normal forms the compactness of the model rises, compared with the bracket iorms the regularity of the model will be higher. Because of the compactness of the model the amount of computation needed for test generation reduces, the tests themselves will not be redundant. Because of the regularity of the model the algorithms of test generation will be simplified.

The general idea of the method lies in the test generation to check the paths of the given circuit. The conditions when test pairs will not be sufficient are given. For such cases test groups must be generated, consisting of three or more single tests. The method to find such test groups will be given. Some theorems will be proved to give the conditions of the test compieteness. 